\title{
Role of CXCL12, TP53 and CYP1A1 gene polymorphisms in susceptibility to pediatric acute lymphoblastic leukemia
}

\author{
ELENI KAMPOURAKI ${ }^{1}$, MARILENA LOUROU ${ }^{1}$, MARIA I. ZERVOU ${ }^{2}$, EVANGELIA-DIMITRA AMPAZOGLOU ${ }^{1}$, \\ EMMANUEL YACHNAKIS ${ }^{3}$, NIKOLAOS KATZILAKIS ${ }^{1}$, GEORGE N. GOULIELMOS ${ }^{2}$ and EFTICHIA STIAKAKI ${ }^{1}$ \\ ${ }^{1}$ Department of Pediatric Hematology-Oncology, University Hospital of Heraklion and Laboratory of \\ Blood Diseases and Childhood Cancer Biology, Medical School, University of Crete; \\ ${ }^{2}$ Section of Molecular Pathology and Human Genetics, Department of Internal Medicine, Medical School of Crete; \\ ${ }^{3}$ Laboratory of Bio-Medical Data Analyses, Digital Applications and Interdisciplinary Approaches, \\ University of Crete, 71003 Heraklion, Greece
}

Received March 22, 2021; Accepted May 26, 2021

DOI: $10.3892 / \mathrm{ol} .2021 .12920$

\begin{abstract}
Acute lymphoblastic leukemia (ALL) is the most common type of childhood leukemia and represents one third of all pediatric malignancies. Epidemiological studies have shown that various genetic factors play a crucial role in leukemogenesis. Recent genetic association studies on cancer risk have focused on the effects of single-nucleotide polymorphisms (SNPs) in genes that regulate inflammation and tumor suppression, such as chemokines, TP53 and cytochrome P450s (CYPs). Genetic polymorphisms in the 3' untranslated region of the C-X-C motif chemokine ligand 12 (CXCL12; rs1801157) and TP53 (rs1042522) genes have been suggested to influence the risk of ALL in children, while other studies have indicated an association between the CYP1 subfamily A member 1 $(C Y P 1 A 1)^{*} 2 C$ (rs1048943) allele and leukemia risk. The aim of the present study was to investigate the possible association of rs1801157 (CXCL12), rs1042522 (TP53) and rs1048943 (CYPIA1 $2 C$ ) SNPs with an increased susceptibility of developing ALL. These SNPs were analyzed in 86 children or adolescent patients with ALL and 125 control subjects by PCR-restriction fragment length polymorphism and allelic-specific chain reaction techniques. A higher frequency of $C Y P 1 A 1^{*} 2 C$ heterozygotes and TP53 rare homozygotes, which include the proline (Pro)/Pro genotype, was observed among children with ALL and control subjects, whereas no significant differences were observed for the CXCL12 SNP. Furthermore, the analysis of various allelic combinations of the aforementioned gene polymorphisms demonstrated a
\end{abstract}

Correspondence to: Professor Eftichia Stiakaki, Department of Pediatric Hematology-Oncology, University Hospital of Heraklion and Laboratory of Blood Diseases and Childhood Cancer Biology, Medical School, University of Crete, 71003 Heraklion, Greece E-mail: efstel@med.uoc.gr

Key words: acute lymphoblastic leukemia, pediatric, polymorphisms, CXCL12, TP53, CYPIA1 markedly increased risk of developing ALL in children. In conclusion, the present study demonstrated that there was a strong association between $C Y P 1 A 1^{*} 2 C$ heterozygotes, as well as the TP53 Pro/Pro genotype, and an increased susceptibility for pediatric ALL in Caucasians.

\section{Introduction}

Acute lymphoblastic leukemia (ALL) is the most common type of childhood leukemia. It accounts for one third of all cancers diagnosed in children under 15 years (1). High survival rates are achieved with the use of contemporary risk-directed therapy. Previous studies have reported 5-year event-free survival rates $>80 \%$ and overall survival rates $>90 \%$ in ALL $(2,3)$. For those children, life-long follow-up is indispensable for observing late effects on learning (neurocognitive effects), physical and psychological development (4). However, a number of children relapse, which has been estimated as a $<10 \%$ relapse rate in several clinical trials (5). In these cases, the outcome remains poor. Epidemiological studies that examined possible risk factors for acute leukemia have shown that inherited genetic variants can play an important role in leukemogenesis (6). Significant heterogeneity has been found in certain genetic alterations that may drive tumorigenesis, such as copy number alterations, structural alterations, gene fusion events and rare germline mutations $(2,7)$.

Recent gene association studies that examined cancer risk have focused on the effects of single-nucleotide polymorphisms (SNPs) of genes involved in the regulation of inflammation and tumor suppression, such as chemokines, TP53 and cytochrome P450s (CYPs) $(8,9)$. Chemokines induce the migration of endothelial and tumor cells and consequently influence distal metastasis (10). C-X-C motif chemokine ligand 12 (CXCL12) is a chemokine expressed in various tumors, which binds to its receptor $\mathrm{C}-\mathrm{X}-\mathrm{C}$ motif chemokine receptor 4 and is considered to play an important role in tumor growth and dissemination (11). The rs1801157 SNP in this gene has been previously investigated regarding the disease pathogenesis. Specifically, this polymorphism is located in the $3^{\prime}$ untranslated region and in combination with a polymor- 
phism of TP53 at codon 72 (rs1042522) may influence the risk of developing ALL in children (12). The aforementioned polymorphism, which is the most widely studied polymorphism of TP53, refers to a $\mathrm{G}$ to $\mathrm{C}$ transversion in exon 4 at codon 72 and results in the three following genotypes: Arginine/Arginine (Arg/Arg), Arginine/Proline (Arg/Pro) and Proline/Proline (Pro/Pro). It has been shown that the Arg allele (major allele) predominates over the Pro allele (13). TP53 is a tumor suppressor protein that is responsible for the maintenance of genome stability and is considered to weaken the migration of cancer cells (14). Several studies have focused on the role of TP53 polymorphisms as susceptibility factors for the development of malignant diseases (15).

In addition, previous studies have demonstrated a possible association between SNPs of the CYP1 subfamily A member 1 (CYPlAl) gene and an increased risk of leukemia. The CYP1A1 gene encodes for CYP1A1, which is a xenobiotic metabolizing enzyme involved in phase I metabolism. It is responsible for the metabolism of several xenobiotic and endogenous compounds to potential carcinogens. It has been confirmed that CYPIAl (as well as CYP1 subfamily B member 1 and other members of CYPs) metabolize polycyclic aromatic hydrocarbons, which are well-known environmental procarcinogens that may induce tumorigenesis following their activation (16). Moreover, certain gene polymorphisms have been reported to alter the catalytic activity of this enzyme. As a consequence, its ability to metabolize carcinogens and other mutagens may be influenced (17). More specifically, the rs1048943 polymorphism of the CYP1Al gene, corresponding to an $\mathrm{A}$ to $\mathrm{G}$ nucleotide change at position 2,455 , has been reported to be a risk factor of ALL in Caucasians (18). The same SNP has been shown to increase the risk for the development of breast, prostate and cervical cancers (19-21). The rs1048943 polymoprhism has been shown to increase the risk of breast cancer in women exposed to PCBs, which are organochloride compounds that can induce the expression of CYPs (19).

To date, the associations between genotypic or allelic combinations of CXCL12, CYPIA1 or TP53 gene polymorphisms with ALL have not been investigated in Greek populations. Therefore, the present study aimed to investigate the plausible association between polymorphisms of $C Y P 1 A 1$, CXCL12 and TP53 genes and the risk of developing ALL in children and adolescent patients from the island of Crete, Greece.

\section{Materials and methods}

Study population. The case group consisted of 86 patients, children and adolescents (51 males and 35 females), which were diagnosed with ALL at the Department of Pediatric Hematology-Oncology of the University Hospital of Heraklion (Crete) from 1990 to January 2018. The youngest age at diagnosis was 9 months and the oldest age at diagnosis was 17 years and 9 months (Tables SI and SII). The control group consisted of 105 adult blood donors (age range between 18 and 60 years) and 20 children (10 males and 10 females, age range between 9 months and 17 years 9 months) from the same region of patients. Therefore, the control group consisted of healthy individuals, free of neoplasia, which were not hospi- talized. The study protocol was approved by the Institutional Committee of the University Hospital of Heraklion (approval no. 263/6/24-03-2016) and signed informed consent was provided by the patients, the parents/guardians and the healthy control subjects.

Analysis of gene polymorphisms. Whole blood was collected in ethylene diaminetetraacetic acid-containing tubes. Genomic DNA was isolated from peripheral blood leukocytes using the Wizard ${ }^{\circledR}$ Genomic DNA Purification kit (Promega Corporation) and the DNA purification kit (Invitrogen; Thermo Fischer Scientific, Inc.) according to the manufacturer's instructions. The extracted DNA was quantified by spectrophotometry and stored at $-20^{\circ} \mathrm{C}$. The amplification and genotyping for the CXCL12 rs1801157, TP53 (codon 72) rs1042522 and CYP1A1 $2 C$ rs1048943 SNPs was performed using restriction fragment length polymorphism-based approaches as previously described $(12,22)$. The amplification of the genomic fragments harboring the polymorphic sites was carried out using a Taq polymerase provided by Invitrogen; Thermo Fisher Scientific, Inc. The analysis of the rs1801157 CXCL12 SNP was performed using forward 5'-CAG TCA ACC TGG GCA AAG CC-3' and the reverse 5'-CCT GAG AGT CCT TTT GCG GG primers in order to generate a 293-base pair (bp) fragment. For genotyping purposes, the 293-bp product was digested with the MspI restriction enzyme (New England BioLabs, Inc). The latter specifically digests DNA amplified from the allele G into 193-bp and 100-bp fragments (12). Moreover, to analyze the TP53 rs1042522 SNP, a protocol previously described by de Lourdes Perim et al (12) was used with minor modifications. The following primers were used for the amplification of Pro and Arg alleles, respectively, which represent the variants of the TP53 rs1042522 gene polymorphism under study: Forward, 5'-GCC AGA GGC TGC TCC CCC-3' and 5'-TCC CCC TTG CCG TCC CAA-3' and reverse, 5'-CGT GCA AGT CAC AGA CTT-3' and 5'-TCC CCC TTG CCG TCC CAA-3'. Aiming to genotype the 'Pro' allele, DNA was amplified using an initial denaturation step of $94^{\circ} \mathrm{C}$ for $3 \mathrm{~min}$, followed by 35 cycles at $94^{\circ} \mathrm{C}$ for $30 \mathrm{sec}, 61^{\circ} \mathrm{C}$ for $30 \mathrm{sec}, 72^{\circ} \mathrm{C}$ for $14 \mathrm{sec}$ and a final elongation step at $72^{\circ} \mathrm{C}$ for $5 \mathrm{~min}$. Moreover, with regard to the genotyping of the 'Arg' allele, DNA was amplified using an initial denaturation step of $94^{\circ} \mathrm{C}$ for $3 \mathrm{~min}$, followed by 35 cycles at $94^{\circ} \mathrm{C}$ for $30 \mathrm{sec}, 61^{\circ} \mathrm{C}$ for $30 \mathrm{sec}$ and $72^{\circ} \mathrm{C}$ for $30 \mathrm{sec}$. A final elongation step was used at $72^{\circ} \mathrm{C}$ for $10 \mathrm{~min}$. The TP53 Pro allele yielded a 178-bp product, while the Arg allele a 136-bp PCR product. Furthermore, to analyze the $C Y P 1 A 1^{*} 2 C$ rs1048943 SNP, a similar protocol to that reported by Razmkhah et al (22) was used. The forward 5'-CTG TCT CCC TCT GGT TAC AGG AAG C-3' and the reverse 5'-TTC CAC CCG TTG CAG CAG GAT AGC C-3' primers were used to generate a 204-bp PCR product. Genotyping was performed by digesting the 204-bp PCR product with the BsrDI restriction enzyme (New England BioLabs, Inc.), which specifically digests DNA amplified from the allele A into 149-bp and 55-bp fragments (22). All PCR products were analyzed using $2.5 \%$ agarose gel electrophoresis. The detection was performed using ethidium bromide and the band size was compared with a molecular weight marker. The genotypes were scored in a blinded study design and analysis of all ambiguous samples was repeated. Moreover, $10 \%$ of the samples were amplified twice for ensuring the accuracy of the results. 
Table I. Genotype rates of CYP1A1, CXCL12 and TP53 for controls and patients with ALL.

\begin{tabular}{|c|c|c|c|c|}
\hline \multirow[b]{2}{*}{ Gene } & \multicolumn{3}{|c|}{ Participants } & \multirow[b]{2}{*}{ Statistical analysis } \\
\hline & 0 & 1 & Total & \\
\hline CYPIAl & & & & $\chi^{2}=16.036 ; \mathrm{df}=2 ; \mathrm{P}<0.001^{\mathrm{a}}$ \\
\hline AA & 105 & 51 & 156 & \\
\hline $\mathrm{AG}$ & 19 & 33 & 52 & \\
\hline GG & 1 & 0 & 1 & \\
\hline Total & 125 & 84 & 209 & \\
\hline CXCL12 & & & & $\chi^{2}=4.154 ; \mathrm{df}=2 ; \mathrm{P}=0.125$ \\
\hline AA & 16 & 9 & 25 & \\
\hline $\mathrm{AG}$ & 55 & 50 & 105 & \\
\hline GG & 49 & 24 & 73 & \\
\hline Total & 120 & 83 & 203 & \\
\hline TP53 & & & & $\chi^{2}=4.565 ; \mathrm{df}=2 ; \mathrm{P}=0.102$ \\
\hline Arg/Arg & 64 & 35 & 99 & \\
\hline Arg/Pro & 47 & 37 & 84 & \\
\hline Pro/Pro & 10 & 14 & 24 & \\
\hline Total & 121 & 86 & 207 & \\
\hline
\end{tabular}

$\chi^{2}$ was performed for the analysis of the $C Y P 1 A 1, C X C L 12$ and TP53 groups. ${ }^{\text {a }}<0.05 .0$, control participants; 1 , patients with ALL; ALL, acute lymphoblastic leukemia; CXCL12, C-X-C motif chemokine ligand 12; CYP1A1, CYP1 subfamily A member 1; Arg, Arginine; Pro, Proline; AA, wild-type genotype; AG, heterozygous for the major allele; GG, homozygous for the minor allele; df, degrees of freedom.

Statistical analysis. Data and statistical analyses were conducted using SPSS v25 software (IBM Corp.) and a set of Microsoft Excel (Microsoft Corporation) applications developed in the laboratory of Bio-Medical Data Analyses, Digital Applications and Interdisciplinary Approaches in Medical School of Crete (Heraklion, Greece). $\mathrm{P}<0.05$ was considered to indicate a statistically significant difference. Chi-square and Fischer's exact tests were performed for data analysis. $2 \times 2$ and $2 \times 3$ contingency tables were produced from the gene frequencies observed in control subjects and patients with ALL. Based on these tables, genotype percentages and odds ratios with the corresponding $95 \%$ confidence intervals were calculated. The wild-type (AA) genotype (major genotype Arg/Arg for TP53) was considered as the reference (OR, 1.0) for the $2 \times 3$ tables.

\section{Results}

Genotypes of patient and control populations. A total of 84 patients (49 males, 35 females) and 125 controls were genotyped for the presence of the rs1048943 polymorphism of the CYPlAl gene, whereas 83 patients (49 males, 34 females) and 120 controls were genotyped for the presence of the rs 1801157 polymorphism of the CXCL12 gene, and 86 patients (51 males, 35 females) and 121 controls were genotyped for the presence of the rs1042522 polymorphism of the TP53 gene. For unknown technical reasons, not all samples were genotyped for the three SNPs examined.

Analyses conducted for CYP1A1, CXCL12 and TP53 gene polymorphisms. In the present study, two analyses were conducted for the investigations of the SNPs. Initially, the following genotypes were analyzed: i) AA, homozygous for the major allele of TP53; ii) AG, heterozygous for the major allele of TP53; and iii) GG, homozygous for the minor allele of TP53. These corresponded to the Arg/Arg, Arg/Pro and Pro/Pro variants, respectively ( 0 and 1 in Table I denote control and patients, respectively). The second analysis examined the presence of a minor allele at least in the heterozygous state (non-AA or non-Arg/Arg for TP53; Table II). The AG genotype of the rs1048943 SNP of CYPIAl was associated with an increased risk of developing ALL $(\mathrm{P}<0.001)$. No associations were detected between the incidence of ALL and the presence of rs1801157 or rs1042522 polymorphisms.

Analyses of combinations of genepolymorphisms. Furthermore, the polymorphisms of the genes CYP1A1, CXCL12 and TP53 were assessed in pairs and altogether as three. The following convention was adopted: In the combination (pair) CYP1A1 + CXCL12, the abbreviation AGAA corresponded to AG for CYP1A1 and AA for CXCL12. Table III, part A, demonstrates the relative risks and odds ratios for $C Y P 1 A 1$, CXCL12 and TP53 (in reference to AA) as well as their combinations in pairs (Table III, part B, with reference to AAAA) and for the set of all three (Table III, part C, with reference to AAAA, Arg/Arg). An individual carrying the non-AA genotype for CYP1A1 (carrying G allele either in homozygosity or heterozygosity) exhibited a 3.397-fold increased probability of developing ALL compared with a subject carrying the AA genotype for the CYPIA1 gene (Table III, part A; OR, 3.397; 95\% CI, 1.776-6.497). The differences were statistically significant. Furthermore, the risk ratio for AG regarding the CYPIAl 
Table II. Rates of controls and patients with ALL not carrying the polymorphism (wild-type, AA or Arg/Arg) and rates of those carrying the polymorphism (non-AA or nonArg/Arg).

\begin{tabular}{|c|c|c|c|c|}
\hline \multirow[b]{2}{*}{ Gene } & \multicolumn{3}{|c|}{ Participants } & \multirow[b]{2}{*}{ Statistical analysis } \\
\hline & 0 & 1 & Total & \\
\hline CYPIAl & & & & $\chi^{2}=14.392 ; \mathrm{df}=1 ; \mathrm{P}<0.001^{\mathrm{a}}$ \\
\hline $\mathrm{AA}$ & 105 & 51 & 156 & \\
\hline Non-AA & 20 & 33 & 53 & \\
\hline Total & 125 & 84 & 209 & \\
\hline CXCL12 & & & & $\chi^{2}=0.282 ; \mathrm{df}=1 ; \mathrm{P}=0.596$ \\
\hline $\mathrm{AA}$ & 16 & 9 & 25 & \\
\hline Non-AA & 104 & 74 & 178 & \\
\hline Total & 120 & 83 & 203 & \\
\hline TP53 & & & & $\chi^{2}=2.996 ; \mathrm{df}=1 ; \mathrm{P}=0.083$ \\
\hline Arg/Arg & 64 & 35 & 99 & \\
\hline Non-Arg/Arg & 57 & 51 & 108 & \\
\hline Total & 121 & 86 & 207 & \\
\hline
\end{tabular}

$\chi^{2}$ was performed for the analysis of the $C Y P 1 A 1, C X C L 12$ and TP53 groups. ${ }^{\mathrm{a} P}<0.05 .0$, control participants; 1 , patients with ALL; ALL, acute lymphoblastic leukemia; CXCL12, C-X-C motif chemokine ligand 12; CYP1A1, CYP1 subfamily A member 1; Arg, Arginine; AA, wild-type genotype; df, degrees of freedom; Non-, presence of a minor allele at least in the heterozygous state.

gene polymorphism was 2.564 compared with that for the AA genotype. This finding indicated that the AG genotype was associated with a 2.564-fold higher risk of developing ALL compared with that of the AA genotype. Therefore, a significant association was noted between the AG genotype and the incidence of ALL (OR, 3.5759; 95\% CI, 1.8553-6.8918). By contrast, the risk ratio for $\mathrm{GG}$ was 0.6589 compared with that of AA, whereas the results were not significantly different. Due to the extremely low frequency of the GG genotype, a supplementary analysis was conducted using Fischer's exact test with the same results, as provided in Table SIII. In addition, the data indicated that a subject with the TP53 Pro/Pro genotype exhibited a 2.56-fold increased probability of developing ALL compared with an individual of the TP53 Arg/Arg genotype (OR, 2.5600; 95\% CI, 1.0303-6.3607). The differences were statistically significant.

Combining gene polymorphisms in pairs. The subjects with the CYP1A1 AG and TP53 Arg/Pro genotypes exhibited 3.14-fold higher risk of developing ALL compared with those who carried the wild-type variant for both genes (OR, 4.6184; 95\% C, 1.6584-12.8620; Table III, part B). Moreover, an individual with CYP1A1 AG and CXCL12 GG genotypes exhibited a 8.29-fold higher risk of developing ALL compared with a subject that carried the AA genotype for both gene polymorphisms (OR, 11.3684; 95\% CI, 2.2156-58.3335). An individual with CYP1A1 AG and CXCL12 AG genotype demonstrated a 1.9-fold higher risk of developing ALL compared with a subject who was homozygous for the major allele $\mathrm{G}$ for both polymorphisms (OR, 5.4000; 95\% CI, 1.6899-17.2557).

Combination of all three gene polymorphisms. An individual with CYP1A1 AG, CXCL12 AG and TP53 Pro/Pro genotypes exhibited 11.2-fold higher risk of developing ALL compared with that of a subject with an AA genotype for CYP1Al and CXCL12 and an Arg/Arg genotype for TP53 (Table III, part C; OR, 11.2000; 95\% CI, 1.0421-120.3681). These comparisons were significantly different.

\section{Discussion}

In the present study, the data indicated that the $C Y P 1 A 1^{*} 2 C$ AG genotype was associated with an increased susceptibility of developing ALL compared with the AA genotype. To the best of our knowledge, this is the first report that demonstrated an association of $C Y P 1 A 1^{*} 2 C$ heterozygotes with an increased risk of developing ALL in Caucasian children. Although, the association between $C Y P 1 A 1$ polymorphisms and leukemia has been studied extensively, the results are contradictory. The fact that $C Y P I A I^{*} 2 C$ increases the risk for developing leukemia was revealed by a meta-analysis conducted by Han et al (17). This analysis indicated that the risk for leukemia was increased in adults (moderate cumulative evidence) but not in children (weak cumulative evidence). The meta-analysis conducted by Lu et al (18) confirmed that the CYP1A1 A2455G polymorphism increased the risk of developing ALL in Caucasians. Furthermore, a study conducted by Joseph et al (23) further indicated that $C Y P 1 A 1^{*} 2 C$ was associated with an increased risk of developing childhood ALL in Indian patients. However, the present study demonstrated that the homozygous variant was associated with an increased risk of developing ALL (23).

The results of the present study are also in accordance with the findings reported by Swinney et al (24), who conducted a study including Caucasian, Hispanic and African-American children. The authors revealed that $C Y P 1 A 1^{*} 2 C$ was associated with an increased risk of developing ALL, whereas following stratification, the risk was statistically significant only in children with Hispanic ancestry (24). In contrast to 
Table III. RR and OR analyses for CYP1A1, CXCL12 and TP53 alone, in pairs and in a set of all three.

A, CYP1A1, CXCL12 and TP53

\begin{tabular}{|c|c|c|c|c|c|c|c|c|}
\hline \multirow[b]{2}{*}{ Genes } & \multicolumn{4}{|c|}{$\mathrm{RR}$} & \multicolumn{4}{|c|}{ OR } \\
\hline & RR value & $95 \% \mathrm{CI}$ & $Z$ value & P-value & OR value & $95 \% \mathrm{CI}$ & $\mathrm{Z}$ value & P-value \\
\hline CYPIA1 non-AA to AA & 2.4554 & $1.5168-3.9748$ & 3.655 & $0.0003^{\mathrm{a}}$ & 3.3971 & $1.7763-6.4967$ & 3.697 & $0.0002^{\mathrm{a}}$ \\
\hline$C Y P 1 A 1 \mathrm{AG}$ to AA & 2.5639 & $1.5679-4.1927$ & 3.752 & $0.0002^{\mathrm{a}}$ & 3.5759 & $1.8553-6.8918$ & 3.806 & $0.0001^{\mathrm{a}}$ \\
\hline$C Y P 1 A 1 \mathrm{GG}$ to $\mathrm{AA}$ & 0.6859 & $0.0284-16.5510$ & 0.232 & 0.8164 & 0.6828 & $0.0273-17.0556$ & 60.232 & 0.8163 \\
\hline CXCL12 non-AA to AA & 1.0287 & $0.9283-1.1400$ & 0.540 & 0.5889 & 1.2650 & $0.5303-3.0174$ & 0.530 & 0.5962 \\
\hline CXCL12 AG to AA & 1.0940 & $0.9269-1.2912$ & 1.063 & 0.2880 & 1.6162 & $0.6558-3.9831$ & 1.043 & 0.2969 \\
\hline CXCL12 GG to AA & 0.9647 & $0.7507-1.2399$ & 0.280 & 0.7792 & 0.8707 & $0.3362-2.2550$ & 0.285 & 0.7756 \\
\hline TP53 non-ArgArg to ArgArg & 1.2589 & $0.9731-1.6286$ & 1.752 & 0.0797 & 1.6361 & $0.9355-2.8615$ & 1.726 & 0.0843 \\
\hline TP53 ArgPro to ArgArg & 1.2137 & $0.8880-1.6587$ & 1.215 & 0.2244 & 1.4395 & $0.7931-2.6128$ & 1.198 & 0.2310 \\
\hline TP53 ProPro to ArgArg & 2.1143 & $1.0222-4.3733$ & 2.019 & $0.0435^{\mathrm{a}}$ & 2.5600 & $1.0303-6.3607$ & 2.024 & $0.0429^{\mathrm{a}}$ \\
\hline
\end{tabular}

B, CYP1A1, CXCL12 and TP53 combinations in pairs

\begin{tabular}{|c|c|c|c|c|c|c|c|c|}
\hline \multirow[b]{2}{*}{ Genes } & \multicolumn{4}{|c|}{ RR } & \multicolumn{4}{|c|}{ OR } \\
\hline & RR value & $95 \% \mathrm{CI}$ & $\mathrm{Z}$ value & P-value & OR value & $95 \% \mathrm{CI}$ & $\mathrm{Z}$ value & P-value \\
\hline $\begin{array}{l}C Y P 1 A 1 C X C L 12 \\
\text { non-AAAA to AAAA }\end{array}$ & 1.0378 & $0.9460-1.1384$ & 0.785 & 0.4326 & 1.4476 & $0.5576-3.7586$ & 0.760 & 0.4473 \\
\hline CYP1A1CXCL12 AAAG to AAAA & 1.0106 & $0.7910-1.2912$ & 0.084 & 0.9327 & 1.0455 & $0.3702-2.9520$ & 0.084 & 0.9331 \\
\hline CYP1A1CXCL12 AAGG to AAAA & 0.9937 & $0.7575-1.3035$ & 0.046 & 0.9635 & 0.9756 & $0.3404-2.7966$ & 0.046 & 0.9633 \\
\hline CYP1A1CXCL12 AGAA to AAAA & 1.7778 & $0.2993-10.5605$ & 0.633 & 0.5268 & 2.0000 & $0.2307-17.3391$ & 0.629 & 0.5293 \\
\hline CYP1A1CXCL12 AGAG to AAAA & 1.9059 & $1.1521-3.1529$ & 2.511 & $0.0120^{\mathrm{a}}$ & 5.4000 & $1.6899-17.2557$ & 2.845 & $0.0044^{\mathrm{a}}$ \\
\hline CYP1A1CXCL12 AGGG to AAAA & 1.0909 & $0.4060-2.9313$ & 0.173 & 0.8630 & 1.1429 & $0.2482-5.2633$ & 0.171 & 0.8639 \\
\hline CYP1A1CXCL12 GGGG to AAAA & 0.6667 & $0.0305-14.5942$ & 0.258 & 0.7968 & 0.6444 & $0.0233-17.8229$ & 0.259 & 0.7953 \\
\hline CYP1A1TP53 non-AAArgArg & 1.3828 & $1.1203-1.7068$ & 3.018 & $0.0025^{\mathrm{a}}$ & 2.5313 & $1.3440-4.7672$ & 2.875 & $0.0040^{\mathrm{a}}$ \\
\hline $\begin{array}{l}\text { CYP1A1TP53 AAArgPro } \\
\text { to AAArgArg }\end{array}$ & 1.3258 & $0.9183-1.9142$ & 1.505 & 0.1323 & 1.7202 & $0.8242-3.5905$ & 1.445 & 0.1485 \\
\hline $\begin{array}{l}\text { CYP1A1TP53 AAProPro } \\
\text { to AAArgArg }\end{array}$ & 1.8155 & $0.6379-5.1672$ & 1.117 & 0.2638 & 2.0301 & $0.5752-7.1650$ & 1.100 & 0.2711 \\
\hline $\begin{array}{l}\text { CYP1A1TP53 AGArgArg } \\
\text { to AAArgArg }\end{array}$ & 2.2963 & $0.9624-5.4787$ & 1.874 & 0.0610 & 2.8421 & $0.9360-8.6303$ & 1.843 & 0.0653 \\
\hline $\begin{array}{l}\text { CYP1A1TP53 AGArgPro } \\
\text { to AAArgArg }\end{array}$ & 3.1484 & $1.4570-6.8034$ & 2.917 & $0.0035^{\mathrm{a}}$ & 4.6184 & $1.6584-12.8620$ & 2.928 & $0.0034^{\mathrm{a}}$ \\
\hline CYP1A1CXCL12 AGGG to AAAA & 8.2963 & $1.8887-36.4417$ & 2.802 & $0.0051^{\mathrm{a}}$ & 11.3684 & $2.2156-58.3335$ & 2.913 & $0.0036^{\mathrm{a}}$ \\
\hline $\begin{array}{l}\text { CYP1A1TP53 GGArgArg } \\
\text { to AAArgArg }\end{array}$ & 0.9333 & $0.0396-21.9922$ & 0.043 & 0.9659 & 0.9316 & $0.0364-23.8398$ & 0.043 & 0.9658 \\
\hline $\begin{array}{l}\text { CXCL12TP53 non-AAArgArg } \\
\text { to AAArgArg }\end{array}$ & 0.9989 & $0.9168-1.0882$ & 0.026 & 0.9791 & 0.9857 & $0.3358-2.8932$ & 0.026 & 0.9791 \\
\hline $\begin{array}{l}\text { CXCL12TP53 AAArgPro } \\
\text { to AAArgArg }\end{array}$ & 0.4000 & $0.0571-2.8001$ & 0.923 & 0.3561 & 0.3000 & $0.0277-3.2501$ & 0.990 & 0.3220 \\
\hline $\begin{array}{l}\text { CXCL12TP53 AAProPro } \\
\text { to AAArgArg }\end{array}$ & 0.7857 & $0.0866-7.1304$ & 0.214 & 0.8303 & 0.7500 & $0.0550-10.2333$ & 0.216 & 0.8292 \\
\hline $\begin{array}{l}\text { CXCL12TP53 AGArgArg } \\
\text { to AAArgArg }\end{array}$ & 0.9123 & $0.6371-1.3063$ & 0.501 & 0.6162 & 0.7222 & $0.2118-2.4629$ & 0.520 & 0.6031 \\
\hline $\begin{array}{l}\text { CXCL12TP53 AGArgPro } \\
\text { to AAArgArg }\end{array}$ & 1.1897 & $0.8595-1.6467$ & 1.047 & 0.2951 & 1.9167 & $0.5755-6.3836$ & 1.060 & 0.2892 \\
\hline $\begin{array}{l}\text { CXCL12TP53 AGProPro } \\
\text { to AAArgArg }\end{array}$ & 1.5000 & $0.7122-3.1594$ & 1.067 & 0.2860 & 2.2500 & $0.5221-9.6971$ & 1.088 & 0.2766 \\
\hline CXCL12TP53 GGArgArg & 0.7771 & $0.4730-1.2768$ & 0.995 & 0.3195 & 0.4800 & $0.1303-1.7687$ & 1.103 & 0.2700 \\
\hline
\end{tabular}


Table III. Continued.

B, CYP1A1, CXCL12 and TP53 combinations in pairs

\begin{tabular}{|c|c|c|c|c|c|c|c|c|}
\hline \multirow[b]{2}{*}{ Genes } & \multicolumn{4}{|c|}{ RR } & \multicolumn{4}{|c|}{ OR } \\
\hline & RR value & $95 \% \mathrm{CI}$ & $Z$ value & $\mathrm{P}$-value & OR value & $95 \% \mathrm{CI}$ & $\mathrm{Z}$ value & P-value \\
\hline $\begin{array}{l}\text { CXCL12TP53 GGArgPro } \\
\text { to AAArgArg }\end{array}$ & 0.9382 & $0.6118-1.4389$ & 0.292 & 0.7701 & 0.8250 & $0.2321-2.9325$ & 0.297 & 0.7662 \\
\hline $\begin{array}{l}\text { CXCL12TP53 GGProPro } \\
\text { to AAArgArg }\end{array}$ & 1.8333 & $0.3863-8.7012$ & 0.763 & 0.4455 & 2.2500 & $0.2850-17.7601$ & 0.769 & 0.4417 \\
\hline
\end{tabular}

C, CYP1A1, CXCL12 and TP53 in a set of all three

\begin{tabular}{|c|c|c|c|c|c|c|c|c|}
\hline \multirow[b]{2}{*}{ Genes } & \multicolumn{4}{|c|}{$\mathrm{RR}$} & \multicolumn{4}{|c|}{ OR } \\
\hline & RR value & $95 \% \mathrm{CI}$ & $\mathrm{Z}$ value & P-value & OR value & $95 \% \mathrm{CI}$ & $\mathrm{Z}$ value & P-value \\
\hline $\begin{array}{l}\text { Non-AAAAArgArg to } \\
\text { AAAAArgArg }\end{array}$ & 0.9950 & $0.9214-1.0744$ & 0.129 & 0.8976 & 0.9245 & $0.2822-3.0290$ & 0.130 & 0.8969 \\
\hline AAAAArgPro to AAAAArgArg & 0.1970 & $0.0129-3.0162$ & 1.167 & 0.2432 & 0.1240 & $0.0056-2.7440$ & 1.321 & 0.1864 \\
\hline AAAAProPro to AAAAArgArg & 0.7500 & $0.0859-6.5481$ & 0.260 & 0.7947 & 0.7000 & $0.0489-10.0148$ & 0.263 & 0.7928 \\
\hline AAAGArgArg to AAAAArgArg & 0.7609 & $0.4536-1.2764$ & 1.035 & 0.3005 & 0.4261 & $0.1024-1.7731$ & 1.173 & 0.2409 \\
\hline AAAGArgPro to AAAAArgArg & 1.1053 & $0.7375-1.6564$ & 0.485 & 0.6278 & 1.4000 & $0.3572-5.4874$ & 0.483 & 0.6292 \\
\hline AAAGProPro to AAAAArgArg & 0.4583 & $0.0650-3.2297$ & 0.783 & 0.4336 & 0.3500 & $0.0295-4.1531$ & 0.832 & 0.4055 \\
\hline AAGGArgArg to AAAAArgArg & 0.7689 & $0.4570-1.2939$ & 0.990 & 0.3224 & 0.4455 & $0.1068-1.8586$ & 1.110 & 0.2672 \\
\hline AAGGArgPro to AAAAArgArg & 0.8846 & $0.5324-1.4698$ & 0.473 & 0.6360 & 0.7000 & $0.1680-2.9174$ & 0.490 & 0.6243 \\
\hline AAGGProPro to AAAAArgArg & 3.0000 & $0.3901-23.0726$ & 1.056 & 0.2912 & 4.2000 & $0.3320-53.1253$ & 1.108 & 0.2677 \\
\hline AGAAArgArg to AAAAArgArg & 0.7500 & $0.0859-6.5481$ & 0.260 & 0.7947 & 0.7000 & $0.0489-10.0148$ & 0.263 & 0.7928 \\
\hline AGAAArgPro to AAAAArgArg & 3.4286 & $0.1647-71.3651$ & 0.796 & 0.4263 & 4.0909 & $0.1387-120.6971$ & 0.816 & 0.4146 \\
\hline AGAGArgArg to AAAAArgArg & 1.5000 & $0.5802-3.8779$ & 0.837 & 0.4028 & 2.1000 & $0.3805-11.5894$ & 0.851 & 0.3946 \\
\hline AGAGArgPro to AAAAArgArg & 1.7679 & $0.7378-4.2360$ & 1.278 & 0.2013 & 3.1500 & $0.6083-16.3119$ & 1.368 & 0.1715 \\
\hline AGAGProPro to AAAAArgArg & 4.9231 & $0.7489-32.3638$ & 1.659 & 0.0971 & 11.2000 & $1.0421-120.3681$ & 1.994 & $0.0461^{\mathrm{a}}$ \\
\hline AGGGArgArg to AAAAArgArg & 0.7500 & $0.0859-6.5481$ & 0.260 & 0.7947 & 0.7000 & $0.0489-10.0148$ & 0.263 & 0.7928 \\
\hline AGGGArgPro to AAAAArgArg & 1.0313 & $0.3143-3.3832$ & 0.051 & 0.9595 & 1.0500 & $0.1592-6.9245$ & 0.051 & 0.9596 \\
\hline AGGGProPro to AAAAArgArg & 0.5000 & $0.0242-10.3422$ & 0.448 & 0.6538 & 0.4545 & $0.0154-13.4108$ & 0.457 & 0.6480 \\
\hline GGGGArgArg to AAAAArgArg & 0.5000 & $0.0242-10.3422$ & 0.448 & 0.6538 & 0.4545 & $0.0154-13.4108$ & 0.457 & 0.6480 \\
\hline
\end{tabular}

AA, AAAA and AAAAArgArg were used as reference groups. ${ }^{\mathrm{P}}<0.05$. RR, relative risk; OR, odds ratio; $C X C L 12, \mathrm{C}-\mathrm{X}-\mathrm{C}$ motif chemokine ligand 12; CYP1A1, CYP1 subfamily A member 1; Arg, Arginine; Pro, Proline; AA, wild-type genotype; AG, heterozygous for the major allele; GG, homozygous for the minor allele; Non-, presence of a minor allele at least in the heterozygous state; df, degree of freedom.

these observations, a study performed in an Iranian population indicated no association between $C Y P 1 A 1^{*} 2 C$ and the risk of developing pediatric ALL (22). The aforementioned data suggest the existence of genetic differences between various ethnic and/or racial populations and therefore the study of each polymorphism in different populations may be meaningful.

In addition, individuals with the CYPIAl GG genotype did not appear to have a higher risk for developing ALL in comparison with the controls. A possible explanation could be attributed to the extremely low frequency of GG genotypes in both cases with ALL and controls in the Cretan population. This observation is consistent with previous findings (22).
The association of the rs1801157 (CXCL12) and rs1042522 (TP53) SNPs has been previously examined in a study that enrolled 54 children with ALL and 58 controls of Caucasian individuals living in Brazil. The authors of that study demonstrated a positive association between these polymorphisms and the risk of developing ALL. Moreover, when both risk genotypes were analyzed in combination, a 5-fold increased risk of developing ALL was observed (12). In the present study, the data indicated that the TP53 Pro/Pro genotype was associated with an increased risk of developing ALL compared with that noted in the TP53 Arg/Arg genotype. However, no association was observed between the CXCL12 polymorphism and the incidence of pediatric ALL. In addition, a combination of TP53 and 
CXCL12 polymorphisms did not increase the risk of ALL in the population examined.

To the best of our knowledge, the present study is the first that investigated in detail the association of developing ALL with the presence of various combinations of the CYP1A1*2C (rs1048943), CXCL12 (rs1801157) and TP53 (rs1042522) polymorphisms. The risk of developing pediatric ALL is significantly increased when an individual exhibits the following combinations of genotypes: i) $C Y P 1 A 1 \mathrm{AG}$ and TP53 Arg/Pro; ii) CYPlAl AG and CXCL12 GG; iii) CYPlAl $\mathrm{AG}$ and CXCL12 AG; and iv) CYP1A1 AG, CXCL12 AG and TP53 Pro/Pro.

The main limitation of the present study was the small sample size of the study population, which did not allow the generalization of the interpretation of the results. Additional subjects were not used that were available from other local populations of Greece in order to avoid any disturbance in the genetic homogeneity of the Cretan population. The small population size is a common limitation noted in similar studies. Sweet-Cordero and Biegel (7) reported that the majority of the pediatric genomic studies had enrolled a relatively small number of patients, making it necessary to pool data to increase analytical power. An additional limitation was that the study and the control groups were not sex- and age-matched. It is not certain whether a matched control (child) will develop ALL later in their life. By contrast, it is considered that blood donors are an appropriate population for the control group in order to assess the effects of the SNPs under investigation, since they are healthy, not hospitalized and free of any neoplasia or disease. The decision for the selection of the control population is consistent with other genetic studies that did not enroll age- and sex-matched controls. For example, a study that investigated the association of genetic polymorphisms of folate-specific genes and the risk of developing pediatric ALL included 500 blood bank donors as a control group (25). A definite advantage of the present study was the selection of a genetically and ethnically homogeneous patient cohort and its corresponding control group. Moreover, the low rate of migration, the availability of optimal genealogical and clinical records and the uniform environment contributed to reduced heterogeneity of the population used. Crete (situated at $25^{\circ} \mathrm{E}$ and $35^{\circ} \mathrm{N}$ ) is the largest island in Greece, with $\sim 0.65$ million inhabitants who share the same genetic and cultural background and a common environment.

Significant heterogeneity has been reported in the types of genetic alterations that may drive leukemogenesis, such as copy number alterations, structural alterations (chromoplexy, chromothripsis), gene fusion events and rare germline mutations, such as ETS translocation variant 6 and paired Box 5 (2,7). To the best of our knowledge, a possible association between driver gene mutations and the combinations of the aforementioned SNPs is yet to be reported. Notably, it has been shown that the presence of SNPs in several biological targets, such as cytokines, drug-metabolizing enzymes and tumor suppressor proteins, can be independently involved in leukemogenesis by affecting proliferation, apoptosis and other associated processes (26). However, a complex interaction of several factors, such as recurrent somatic, cytogenetic and molecular alterations, is considered to be associated with predisposing environmental factors (27).
Effective screening methods are required for early detection and prevention of ALL. Accordingly, Wang et al (28) demonstrated that the combination of SNP array analysis with chromosome and fluorescence in situ hybridization (FISH) assays in patients with ALL significantly increased the detection rate for clinically abnormal conditions. It is also deduced that SNP arrays may be an optimal approach to be used in the future as a complementary assay to the existing chromosome and FISH assays. Additional studies utilizing the SNP screening panel are required given that a large number of ALL-associated SNPs remain unidentified (29). The detection of numerous leukemia-associated gene polymorphisms has also been explored by genome wide association studies (29).

The present study demonstrated a strong association between $C Y P 1 A 1^{*} 2 C$ heterozygotes, the TP53 Pro/Pro genotype and an increased susceptibility for pediatric ALL in Caucasians. Additional studies with a larger number of patients and controls are required to support the data presented. Moreover, the results provide a starting point for future research using larger samples for the investigation of the aforementioned genotype combinations that contribute to the increased risk of ALL in the population examined. This may be considered a promising screening tool to evaluate increased ALL risk in children.

\section{Acknowledgements}

Not applicable.

\section{Funding}

The present study was supported by the University of Crete Secretariat Research Committee (grant no. KA 4440)

\section{Availability of data and materials}

The datasets used and/or analyzed during the current study are available from the corresponding author on reasonable request.

\section{Authors' contributions}

EK, ML and ES conceived and designed the study. EK, ML, NK, MIZ, EDA and GNG performed the experiments and obtained the data. EK, MZ, EY, GNG and EDA searched the literature, and analyzed and interpreted the data. EK, ML, EY and NK drafted the manuscript. MIZ, EDA, GNG and ES critically revised the manuscript for important intellectual content. EK and ES confirm the authenticity of all the raw data. All authors have read and approved the final manuscript.

\section{Ethics approval and consent to participate}

Ethical approval was granted by the Institutional Committee of The University Hospital of Heraklion (approval no. 263/6/24-03-2016; Heraklion, Greece). Signed informed consent was provided by the patients, the parents/guardians and the healthy control subjects.

\section{Patient consent for publication}

Not applicable. 


\section{Competing interests}

The authors declare that they have no competing interests.

\section{References}

1. Belson M,Kingsley B and Holmes A: Risk factors for acute leukemia in children: A review. Environ Health Perspect 115: 138-145, 2007.

2. Tasian SK and Hunger SP: Genomic characterization of paediatric acute lymphoblastic leukaemia: An opportunity for precision medicine therapeutics. Br J Haematol 176: 867-882, 2017.

3. Hunger SP and Mullighan CG: Acute Lymphoblastic Leukemia in Children. N Engl J Med 373: 1541-1552, 2015.

4. Mavrea K, Efthymiou V, Katsibardi K, Tsarouhas K, Kanaka-Gantenbein C, Spandidos DA, Chrousos G, Kattamis A and Bacopoulou F: Cognitive function of children and adolescent survivors of acute lymphoblastic leukemia: A meta-analysis. Oncol Lett 21: 262, 2021.

5. Pui CH: Precision medicine in acute lymphoblastic leukemia. Front Med 14: 689-700, 2020.

6. Iacobucci I and Mullighan CG: Genetic basis of acute lymphoblastic leukemia. J Clin Oncol 35: 975-983, 2017.

7. Sweet-Cordero EA and Biegel JA: The genomic landscape of pediatric cancers: Implications for diagnosis and treatment. Science 363: 1170-1175, 2019.

8. Vijayakrishnan $\mathbf{J}$ and Houlston RS: Candidate gene association studies and risk of childhood acute lymphoblastic leukemia: A systematic review and meta-analysis. Haematologica 95: 1405-1414, 2010.

9. Kampouraki E, Goulielmos GN and Stiakaki E: Understanding the role of genetics in childhood acute lymphoblastic leukemia (Review). World Acad Sci J: Jun 16, 2020 (Epub ahead of print).

10. Mishra P, Banerjee D and Ben-Baruch A: Chemokines at the crossroads of tumor-fibroblast interactions that promote malignancy. J Leukoc Biol 89: 31-39, 2011.

11. Müller A, Homey B, Soto H, Ge N, Catron D, Buchanan ME, McClanahan T, Murphy E, Yuan W, Wagner SN, et al: Involvement of chemokine receptors in breast cancer metastasis. Nature 410: 50-56, 2001.

12. de Lourdes Perim A, Guembarovski RL, Oda JM, Lopes LF, Ariza CB, Amarante MK, Fungaro MH, de Oliveira KB and Watanabe MA: CXCL12 and TP53 genetic polymorphisms as markers of susceptibility in a Brazilian children population with acute lymphoblastic leukemia (ALL). Mol Biol Rep 40: 4591-4596, 2013

13. Costa KA and Guillo LA: TP53 codon 72 polymorphism in pigmentary phenotypes. J Biosci 37: 33-39, 2012.

14. Moskovits N, Kalinkovich A, Bar J, Lapidot T and Oren M: p53 Attenuates cancer cell migration and invasion through repression of SDF-1/CXCL12 expression in stromal fibroblasts. Cancer Res 66: 10671-10676, 2006

15. Olivier M, Hollstein M and Hainaut P: TP53 mutations in human cancers: Origins, consequences, and clinical use. Cold Spring Harb Perspect Biol 2: a001008, 2010.

16. Elfaki I, Mir R, Almutairi FM and Duhier FM: Cytochrome P450: Polymorphisms and Roles in Cancer, Diabetes and Atherosclerosis. Asian Pac J Cancer Prev 19: 2057-2070, 2018.

17. Han F, Tan Y, Cui W, Dong L and Li W: Novel insights into etiologies of leukemia: A HuGE review and meta-analysis of CYP1A1 polymorphisms and leukemia risk. Am J Epidemiol 178: 493-507, 2013
18. Lu J, Zhao Q, Zhai YJ, He HR, Yang LH, Gao F, Zhou RS, Zheng $\mathrm{J}$ and Ma XC: Genetic polymorphisms of CYP1A1 and risk of leukemia: A meta-analysis. OncoTargets Ther 8: 2883-2902, 2015

19. Docea AO, Vassilopoulou L, Fragou D, Arsene AL, Fenga C, Kovatsi L, Petrakis D, Rakitskii VN, Nosyrev AE, Izotov BN, et al: CYP polymorphisms and pathological conditions related to chronic exposure to organochlorine pesticides. Toxicol Rep 4: 335-341, 2017.

20. Hoidy WH, Jaber FA and Al-Askari MA: Association of CYP1A1 rs1048943 polymorphism with prostate cancer in Iraqi men patients. Asian Pac J Cancer Prev 20: 3839-3842, 2019.

21. Li S, Li G, Kong F, Liu Z, Li N, Li Y and Guo X: The association of CYP1A1 gene with cervical cancer and additional SNP-SNP interaction in Chinese women. J Clin Lab Anal 30: 1220-1225, 2016.

22. Razmkhah F, Pazhakh V, Zaker F, Atashrazm F and Sheikhi M: Frequency of CYP1A1*2C polymorphism in patients with leukemia in the Iranian population. Lab Med 42: 220-223, 2011.

23. Joseph T, Kusumakumary P, Chacko P, Abraham A and Radhakrishna Pillai M: Genetic polymorphism of CYP1A1, CYP2D6, GSTM1 and GSTT1 and susceptibility to acute lymphoblastic leukaemia in Indian children. Pediatr Blood Cancer 43: 560-567, 2004.

24. Swinney RM, Beuten J, Collier AB III, Chen TT, Winick NJ, Pollock BH and Tomlinson GE: Polymorphisms in CYP1A1 and ethnic-specific susceptibility to acute lymphoblastic leukemia in children. Cancer Epidemiol Biomarkers Prev 20: 1537-1542, 2011.

25. de Jonge R, Tissing WJ, Hooijberg JH, Jansen G, Kaspers GJ, Lindemans J, Peters GJ and Pieters R: Polymorphisms in folate-related genes and risk of pediatric acute lymphoblastic leukemia. Blood 113: 2284-2289, 2009.

26. Asnafi AA, Farshchi N, Khosravi A, Ketabchi N, Behzad MM and Shahrabi S: Significance of genetic polymorphisms in hematological malignancies: Implications of risk factors for prognosis and relapse. Mag Eur Med Oncol 11: 330-344, 2018.

27. Fujita TC, Sousa-Pereira N, Amarante MK and Watanabe MA: Acute lymphoid leukemia etiopathogenesis. Mol Biol Rep 48: 817-822, 2021.

28. Wang Y, Miller S, Roulston D, Bixby D and Shao L: Genome-wide single-nucleotide polymorphism array analysis improves prognostication of acute lymphoblastic leukemia/lymphoma. J Mol Diagn 18: 595-603, 2016.

29. Vijayakrishnan J, Studd J, Broderick P, Kinnersley B, Holroyd A, Law PJ, Kumar R, Allan JM, Harrison CJ, Moorman AV, et al; PRACTICAL Consortium: Genome-wide association study identifies susceptibility loci for B-cell childhood acute lymphoblastic leukemia. Nat Commun 9: 1340, 2018. 